\title{
Tail Ordering Due to Headgroup Hydrogen Bonding Interactions in Surfactant Monolayers at the Water-Oil Interface
}

\author{
Aleksey M. Tikhonov, ${ }^{*} \pitchfork$ Harshit Patel, $\$$ Shekhar Garde, ${ }^{\ddagger}$ and Mark L. Schlossman $*$, \\ Center for Advanced Radiation Sources, University of Chicago, and National Synchrotron Light Source, \\ Beamline X19C, Brookhaven National Laboratory, Upton, New York 11973, Department of Chemical and \\ Biological Engineering, Rensselaer Polytechnic Institute, Troy, New York 12180, and Departments of Physics \\ and Chemistry, University of Illinois, 845 W. Taylor Street, Chicago, Illinois 60607
}

Received: June 30, 2006; In Final Form: August 14, 2006

\begin{abstract}
Interactions between surfactants, and the resultant ordering of surfactant assemblies, can be tuned by the appropriate choice of head- and tailgroups. Detailed studies of the ordering of monolayers of long-chain $n$-alkanoic and $n$-alkanol monolayers at the water-vapor interface have demonstrated that rigid-rod all-trans ordering of the tailgroups is maintained upon replacing the alcohol with a carboxylic acid headgroup. In contrast, at the water-hexane liquid-liquid interface, we demonstrate that substitution of the $-\mathrm{CH}_{2} \mathrm{OH}$ with the $-\mathrm{COOH}$ headgroup produces a major conformational change of the tailgroup from disordered to ordered. This is demonstrated by the electron density profiles of triacontanol $\left(\mathrm{CH}_{3}\left(\mathrm{CH}_{2}\right)_{29} \mathrm{OH}\right)$ and triacontanoic acid $\left(\mathrm{CH}_{3}\left(\mathrm{CH}_{2}\right)_{28} \mathrm{COOH}\right)$ monolayers at the water-hexane interface, as determined by $\mathrm{X}$-ray reflectivity measurements. Molecular dynamics simulations illustrate the presence of hydrogen bonding between the triacontanoic acid headgroups that is likely responsible for the tail ordering. A simple free energy illustrates the interplay between the attractive hydrogen bonding and the ordering of the tailgroup.
\end{abstract}

\section{Introduction}

The development of complex soft materials relies upon understanding the molecular and mesoscopic ordering of surfactant assemblies that often determine the properties of these materials. Molecular ordering within these assemblies can be probed by a variety of scattering and spectroscopic techniques when the surfactants are assembled on solid or liquid surfaces. However, systems containing liquids such as oil and water, which are otherwise immiscible, have internal interfaces at which amphiphilic molecules can assemble. ${ }^{1}$ Probing molecular ordering at these buried interfaces is a more difficult task. ${ }^{2,3}$ Only a limited number of studies of this nature have been reported, and they indicate specifically that surfactant ordering at internal liquid-liquid interfaces is very different than that at liquid surfaces. ${ }^{4-6}$

For example, X-ray studies of the molecular ordering of longchain fluorocarbon $\left(\mathrm{CF}_{3}\left(\mathrm{CF}_{2}\right)_{n}\left(\mathrm{CH}_{2}\right)_{2} \mathrm{OH}, n=7,9\right)$ and hydrocarbon $\left(\mathrm{CH}_{3}\left(\mathrm{CH}_{2}\right)_{m} \mathrm{OH}, m=19,21,23,29\right) n$-alkanol monolayers at water-vapor and water-hexane (liquid-liquid) interfaces highlight interesting differences between structures at these two interfaces..$^{5-7}$ On the water surface, these surfactants form ordered monolayers with rigid-rod all-trans tails for both fluorocarbon and hydrocarbon tail surfactants. ${ }^{5-7}$ Although individual fluorocarbon $n$-alkanols are rigid by nature, the hydrocarbon $n$-alkanols have flexible tails. The formation of

* Corresponding author. E-mail: schloss@uic.edu; phone: 3129968787 fax: 3129969016 (M.L.S.). E-mail: tikhonov@bnl.gov (A.M.T.).

University of Chicago and Brookhaven National Laboratory.

$\doteqdot$ Rensselaer Polytechnic Institute.

$\S$ University of Illinois. rigid-rod monolayers for the surfactants with flexible tails indicates that attractive forces between the tails can overcome any disordering tendency resulting from the flexibility of the alkyl tail. ${ }^{5,6}$ These same surfactants were studied at the waterhexane interface by dissolving them in hexane and probing the monolayer that was spontaneously formed at the interface..$^{5,6}$ Since favorable interactions between the surfactants and hexane allowed them to dissolve, it is sensible to consider the possibility that hexane can mix into the monolayer. However, the inflexible fluorocarbon tails form rigid-rod monolayers and expel the hexane molecules from the interface. ${ }^{7}$ In contrast, the flexible hydrocarbon $n$-alkanol tails are disordered and may allow hexane to penetrate the tail region. 5,6

These comparative measurements reported recently highlight the importance of conformational entropy (arising from tail flexibility) and enthalpic contributions (that contribute to the surfactant solubility) in determining the molecular ordering of the surfactant assembly. The surfactant ordering in the example above is dominated by the tails of the surfactants. If the tails are flexible, then the conformational entropy gained by disordering, combined with favorable interactions with the hexane, produces a liquid monolayer (i.e., with disordered tails). Neither tail flexibility by itself (as for hydrocarbon $n$-alkanols at the water-vapor interface) nor solubility of the surfactant by itself (as for the fluorocarbon $n$-alkanols at the water-hexane interface) is sufficient to produce liquid monolayers.

Although this tail-dominated view of surfactant ordering at the water-oil interface is adequate to qualitatively explain the previous example, it is sensible to expect that strong interactions between the headgroups can also be important. Here, we use $\mathrm{X}$-ray reflectivity measurements to show that triacontanoic acid 


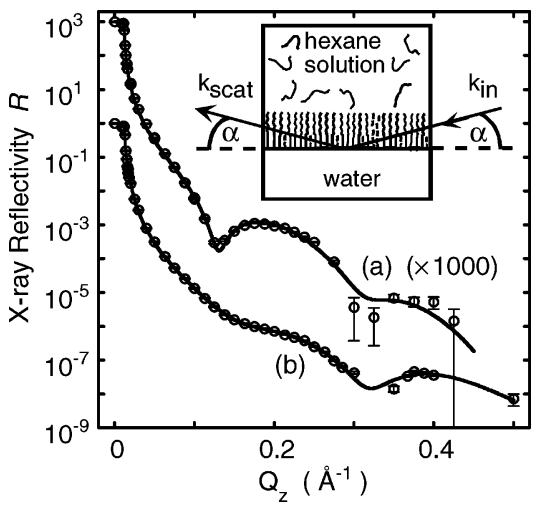

Figure 1. X-ray reflectivity, $R$, as a function of the wave vector transfer normal to the interface, $Q_{z}$, for triacontanoic acid $\left(\mathrm{CH}_{3}\left(\mathrm{CH}_{2}\right)_{28} \mathrm{COOH}\right)$ and triacontanol $\left(\mathrm{CH}_{3}\left(\mathrm{CH}_{2}\right)_{29} \mathrm{OH}\right)$ monolayers at the water-hexane interface $(\mathrm{pH} 2$ water against a $0.2 \mathrm{mmol} / \mathrm{kg}$ solution of triacontanoic acid in hexane, and neutral $\mathrm{pH}$ water against a $0.7 \mathrm{mmol} / \mathrm{kg}$ solution of triacontanol in hexane). (a) Triacontanoic acid monolayers, $T=16.0$ ${ }^{\circ} \mathrm{C}$ (data multiplied by 1000); (b) triacontanol monolayers, $T=24.5$ ${ }^{\circ} \mathrm{C}$. Circles are data, solid lines are fits described in the text. Inset: Geometry of X-ray reflectivity. The X-rays penetrate through the upper bulk phase of hexane solution, then specularly reflect off a nearly planar water-hexane interface (molecules are not to scale in this drawing). The wave vector transfer, $Q=k_{\text {scat }}-k_{\text {in }}$, is normal to the interface (in the $z$-direction) such that $Q_{z}=(4 \pi / \lambda) \sin \alpha$, where $\alpha$ is the angle of incidence and reflection, $\lambda=0.825 \pm 0.002 \AA$ is the $\mathrm{X}$-ray wavelength, and $Q_{x}=Q_{y}=0$ where $x$ and $y$ are in the plane of the interface.

$\left(\mathrm{CH}_{3}\left(\mathrm{CH}_{2}\right)_{28} \mathrm{COOH}\right)$ forms rigid-rod monolayers at the waterhexane interface, even though it differs from triacontanol $\left(\mathrm{CH}_{3}\left(\mathrm{CH}_{2}\right)_{29} \mathrm{OH}\right)$, which forms disordered-tail monolayers, by essentially just one oxygen in the headgroup. Molecular dynamics simulations of these surfactants at the water-hexane interface reveal extensive hydrogen bonding along rows of $-\mathrm{COOH}$ headgroups, but much less hydrogen bonding between the $-\mathrm{CH}_{2} \mathrm{OH}$ groups. Hydrogen bonding is a strong attractive interaction between the headgroups that tips the balance toward surfactant tail ordering in the triacontanoic acid monolayers.

\section{Data and Analysis}

Monolayers of triacontanoic acid and triacontanol were prepared by dissolving them in hexane, then pouring the hexane solution on top of water in a vapor-tight stainless steel sample. ${ }^{7,8}$ The addition of sulfuric acid set the water $\mathrm{pH}$ equal to 2 for the triacontanoic acid sample, and pure water was used for the triacontanol sample. Under these conditions, the headgroups of both surfactants are expected to be uncharged. ${ }^{9,10}$ Alkanol monolayers on the water surface are known to be insensitive to changes in $\mathrm{pH}$ over the range of $2-10 .{ }^{9} \mathrm{X}$-ray reflectivity was measured from this single, large $\left(76 \times 100 \mathrm{~mm}^{2}\right)$, flat interface at beamline X19C of the National Synchrotron Light Source (Brookhaven National Laboratory) with a liquid surface instrument and measurement techniques described in detail elsewhere. ${ }^{7,11}$ The surfactants were studied at temperatures and bulk concentrations at which they form monolayers that fully cover the water-hexane interface. We have previously shown that triacontanol will desorb from the interface into the bulk hexane at a higher temperature. ${ }^{5,6} \mathrm{~A}$ similar phenomena occurs for triacontanoic acid. Although only two data sets are presented here, the conclusions drawn are supported by many data sets for samples at different temperatures and $\mathrm{pH}$. These other data sets for triacontanol have been previously published. ${ }^{5,6}$ Additional data for triacontanoic acid, including the temperature and $\mathrm{pH}$ dependence, will be presented in future publications.

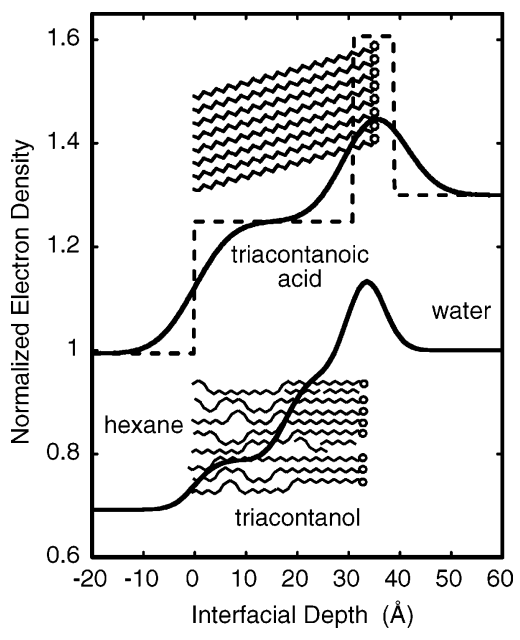

Figure 2. Electron density (normalized to the density for bulk water) as a function of interfacial depth at a water-monolayer-hexane interface for a triacontanoic acid monolayer (top, offset by +0.3 ) and a triacontanol monolayer (bottom). To clearly indicate the layer structure, the dashed line shows the electron density for triacontanoic acid with the interfacial roughness parameter set to zero. The cartoon of molecules is for illustrative purposes only. Long molecules represent the surfactants, and the short molecules within the triacontanol represent hexane. The alkyl tails in the triacontanoic acid monolayer are nearly close-packed, whereas the triacontanol monolayer tails are progressively disordered from a relatively ordered region near the water to a disordered liquid-like region adjacent to bulk hexane.

Figure 1 illustrates $\mathrm{X}$-ray reflectivity measured from triacontanoic acid and triacontanol monolayers at the water-hexane interface. Oscillations in the reflectivity measurements are a result of the coherent interference of X-rays reflected from variations in the electron density with interfacial depth. The two $\mathrm{X}$-ray measurements exhibit quantitative differences. The first minimum in the triacontanoic acid data occurs at a lower wave vector transfer $Q_{z}$ than in the triacontanol data, which indicates that the triacontanoic acid monolayer is thicker. The amplitude of the first two oscillations is larger for the triacontanoic acid data, which usually indicates that the electron density contrast between different interfacial depths is larger.

The oscillations in the data were fitted by a standard procedure using the first Born approximation along with a model for the interfacial profile that consists of two or three layers sandwiched between bulk water and hexane. ${ }^{6,12}$ The interface between each of these layers and an adjacent layer (or bulk) is roughened by capillary waves such that a single roughness parameter describes all the interfaces. The other fitting parameters include the electron density and thickness of each layer.

Figure 2 illustrates the electron density profiles that result from fitting the data in Figure 1, and Table 1 lists the parameters for these fits. The data for the triacontanoic acid monolayers can be fit with a two-layer model. The normalized electron density of 0.95 for the tailgroup layer is comparable to the density in the $\alpha$ or rotator phases of long chain alkanes. ${ }^{13}$ The volume per $-\mathrm{CH}_{2}-$ in the alkane rotator phases varies from 25 to $26 \AA^{3}$, which corresponds to a normalized density of $0.96-$ $0.92 .{ }^{13}$ The rotator phases are solid phases with closely packed all-trans alkyl tails with a small degree of rotational freedom about the long axis.

The headgroup layer has a higher electron density with a maximum value of nearly 1.15 that is in excellent agreement with earlier reflectivity measurements of tetracosanoic acid $\left(\left(\mathrm{CH}_{3}\left(\mathrm{CH}_{2}\right)_{22} \mathrm{COOH}\right)\right)$ at the $\mathrm{pH} 2$ water-vapor interface. ${ }^{14}$ The total thickness of the monolayer, $39 \pm 3 \AA$, is close to the 
TABLE 1: Fitting Parameters

\begin{tabular}{|c|c|c|c|c|c|c|c|c|c|c|c|}
\hline \multirow[b]{2}{*}{ systems } & \multirow[b]{2}{*}{$\begin{array}{c}T^{a} \\
\left({ }^{\circ} \mathrm{C}\right)\end{array}$} & \multirow[b]{2}{*}{$\begin{array}{c}\sigma^{b} \\
(\AA)\end{array}$} & \multicolumn{3}{|c|}{ layer 1} & \multicolumn{2}{|c|}{ layer 2} & \multicolumn{2}{|c|}{ layer 3} & \multirow[b]{2}{*}{$\begin{array}{l}L_{\text {total }} \\
(\AA)\end{array}$} & \multirow[b]{2}{*}{$\begin{array}{l}A_{\mathrm{m}}{ }^{f} \\
\left(\AA^{2}\right)\end{array}$} \\
\hline & & & $\begin{array}{l}L_{1}^{c} \\
(\AA)\end{array}$ & $\rho_{1}^{d}$ & $\rho_{\max }^{e}$ & $\begin{array}{l}L_{2} \\
(\AA)\end{array}$ & $\rho_{2}$ & $\begin{array}{l}L_{3} \\
(\AA)\end{array}$ & $\rho_{3}$ & & \\
\hline $\begin{array}{l}\text { triacontanoic acid } \\
\text { triacontanol }\end{array}$ & $\begin{array}{l}16.0 \\
24.5\end{array}$ & $\begin{array}{l}5.8+0.3 /-1 \\
3.4 \pm 0.4\end{array}$ & $\begin{array}{l}8 \pm 5 \\
4+5 /-2\end{array}$ & $\begin{array}{l}1.3+0.2 /-0.1 \\
1.3 \pm 0.3\end{array}$ & $\begin{array}{l}1.15 \pm 0.01 \\
1.13 \pm 0.01\end{array}$ & $\begin{array}{l}31 \pm 2 \\
13 \pm 2\end{array}$ & $\begin{array}{l}0.95 \pm 0.01 \\
0.95 \pm 0.03\end{array}$ & $\begin{array}{l}\mathrm{n} / \mathrm{a} \\
18 \pm 1\end{array}$ & $\begin{array}{l}\mathrm{n} / \mathrm{a} \\
0.79 \pm 0.01\end{array}$ & $\begin{array}{l}39 \pm 3 \\
35+4 /-1\end{array}$ & $\begin{array}{l}19 \pm 1 \\
23 \pm 1\end{array}$ \\
\hline
\end{tabular}

${ }^{a} T$-temperature. ${ }^{b} \sigma$ - interfacial roughness. ${ }^{c}$ Layers are ordered as water-headgroup (layer 1)-layer 2-layer 3-hexane; $L$ is the layer thickness. ${ }^{d} \rho_{i}$ is the electron density of layer $i$ normalized to the value of bulk water at the temperature for the measurement (e.g., $0.3337 \mathrm{e}^{-} / \AA^{3}$ at $T=20{ }^{\circ} \mathrm{C}$ ); the normalized hexane density is, for example, 0.694 at $T=16^{\circ} \mathrm{C} .{ }^{e}$ For the headgroup, the maximum electron density as well as the density of layer 1 are given because the density and layer thickness fitting parameters are strongly correlated for this thin layer, but the resultant profile is well determined. ${ }^{f} A_{\mathrm{m}}-$ interfacial area per molecule. ${ }^{6}$

calculated all-trans length of $40.8 \AA(=29 \times 1.27 \AA(\mathrm{C}-\mathrm{C})+$ $1.5 \AA\left(-\mathrm{CH}_{3}\right)+2.5 \AA(-\mathrm{COOH})$ ). Although within the error bars of the calculated value, the slightly smaller experimental value may indicate that the tails are tilted from the interfacial normal by as much as $\theta=\cos ^{-1}(39 / 40.8)=17^{\circ}$, however, the reflectivity is not very sensitive to this tilt.

As previously published, the reflectivity from the triacontanol monolayer cannot be fit by a two-layer model; instead, three layers are required. ${ }^{5,6}$ Two of these layers represent the electron density along the alkyl tail; the third layer represents the region of the $-\mathrm{CH}_{2} \mathrm{OH}$ headgroups. The maximum electron density in the headgroup region, $\rho_{\max }=1.13 \pm 0.01$, is comparable for the triacontanol and triacontanoic acid monolayers. The normalized electron densities for the two layers modeling the tail are $0.95 \pm 0.01$ and $0.79 \pm 0.02$. The value of 0.79 in the part of the tail containing the methyl end group corresponds closely to the value of 0.81 for bulk liquid alkyl tails near their freezing point (bulk measurements yield $29.6 \AA^{3} / \mathrm{CH}_{2}$ ). ${ }^{13}$ Closer to the headgroup, the tail has to be more ordered to yield a normalized electron density of 0.95 , which is the same value as the density of the triacontanoic acid tails. As previously discussed, ${ }^{5,6}$ an arrangement that is quantitatively consistent with our measured densities consists of a well-ordered tail near the $-\mathrm{CH}_{2} \mathrm{OH}$ group that is mixed with hexane (up to $25 \mathrm{vol} \%$ ). The tail becomes progressively more disordered toward the methyl end of the tailgroup.

\section{Discussion}

These results indicate that condensed monolayers of triacontanol are liquid-like with disordered tails, but those of triacontanoic acid are in a solid rotator phase with ordered tails. This conclusion is strengthened by noting that the triacontanol monolayer was studied at a temperature $\left(T=24.5^{\circ} \mathrm{C}\right)$ slightly above the temperature at which the bulk hexane is saturated with triacontanol (as observed by the formation of bulk triacontanol crystallites at $T \approx 22{ }^{\circ} \mathrm{C}$ ). Under these conditions, the triacontanol monolayer at the water-hexane interface will be close to its densest state for that temperature. Our previous studies of several tail lengths of alkanols over a range of temperatures, some of which were carried out very close to the saturation temperature, indicate that the liquid monolayer phase is the densest state. ${ }^{6}$

The dominant intermolecular interactions between the surfactants are the attractive van der Waals interactions between the tails and the attractive or repulsive headgroup interactions. The van der Waals interactions are essentially the same for triacontanol and triacontanoic acid. The carbonyl in the headgroup is the primary difference between these surfactants. It allows for extensive hydrogen bonding between the triacontanoic acid headgroups, as illustrated by a molecular dynamics simulation at the water-hexane interface (Figure 3). The hydrogenbonded headgroups are arranged in linear rows, similar to the

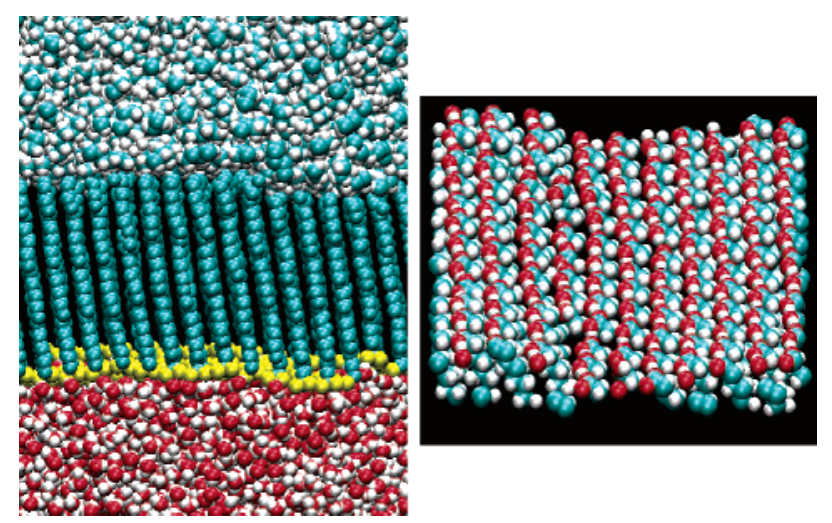

Figure 3. Molecular dynamics simulation of a triacontanoic acid monolayer at the water-hexane interface. Color scheme: $\mathrm{H}-$ white, $\mathrm{C}$ - blue, $\mathrm{O}$ - red, except that headgroups of triacontanoic acid in the left view are yellow. View on the left illustrates the ordered all-trans alkyl tails (from bottom to top: water/triacontanoic acid/hexane). The view on the right, with hexane, water molecules, and most of the surfactant tail removed, illustrates nearly parallel rows of hydrogen bonds between adjacent $-\mathrm{COOH}$ headgroups. However, defects can also be seen, where a row is discontinuous and does not span the entire length. Such a defect also appears to give rise to a small (out of the $x-y$ plane) step in the surface of the surfactants. The simulation cell size was $54.3 \times 57.2 \times 92.0 \AA^{3}$ (normal to the interface) and contained 2720 water molecules, 475 hexane molecules, and 136 triacontanoic acid molecules. Simulations were performed at a constant temperature of $293 \mathrm{~K}$ and a pressure of $1 \mathrm{~atm}$ maintained using a Berendsen thermo and barostat. ${ }^{21}$ Water molecules were represented explicitly using the transferable intermolecular potential 3-point (TIP3P) water model, ${ }^{22}$ hexane molecules and the surfactant molecules were represented explicitly using bond length and angle, torsion parameters, and partial charges from the AMBER force field. ${ }^{23}$ Bonds involving hydrogen atoms were constrained using SHAKE. ${ }^{24}$ Anisotropic pressure coupling was implemented. Periodic boundary conditions were used in all three directions, and the particle mesh Ewald method ${ }^{25}$ with a grid spacing of approximately $1.2 \AA$ was used to calculate electrostatic interactions. A time step of $2 \mathrm{fs}$ was used during a total simulation time of $700 \mathrm{ps}$.

linear H-bonded wires formed by water molecules in hydrophobic environments (e.g., inside carbon nanotubes ${ }^{15}$ ). The linear hydrogen-bonded arrangements were formed in different simulations started with different initial structural arrangements. Furthermore, simulated annealing (heating the surfactant phase to a high-temperature disordered structure, then cooling) reproduces the linear hydrogen-bonded arrangements seen in Figure 3. Also visible in Figure 3 are the triacontanoic acid tailgroups, which are close-packed, essentially all-trans, and slightly tilted $\left(9^{\circ}\right)$. These properties are consistent with our analysis of the $\mathrm{X}$-ray reflectivity data. Although triacontanol headgroups can also hydrogen bond to other headgroups, our molecular dynamics simulations (not shown) indicate that they do not form long continuous chains. Instead, clusters of two to three headgroups are hydrogen-bonded to each other and to water. In addition, slightly less than half of the alkanol headgroups are not hydrogen 
bonded to other headgroups. The tail regions of triacontanol molecules were, however, not completely mixed with hexane over the nanosecond-long simulation times.

The attractive energy gained by hydrogen bonding $(\sim 8 \mathrm{kT}$ per bond) is comparable to the conformational free energy lost ( $\sim 1$ kT per gauche conformation) when a disordered tail becomes all-trans. As discussed elsewhere, literature values lead to an estimate of approximately five gauche conformations in the disordered half of the triacontanol tail. ${ }^{6,16}$ This demonstrates the plausibility of a model in which the presence of the attractive hydrogen bonding brings the surfactants closer together while ordering what would otherwise be a partially ordered tail to an all-trans tail. Note that chain ordering is more likely when the hydrogen bonds link a row of headgroups (as for triacontanoic acid in Figure 3), rather than just bonding isolated pairs (or triplets) of headgroups (as observed in the molecular dynamics simulation of triacontanol), because the ratio of total hydrogen bonding energy to total conformational free energy is greater. In the case of triacontanol monolayers, we find that the bonding geometry and interactions with water destabilizes the formation of rows of H-bonded headgroups.

Alternatively, the effect of the hydrogen bonding can be considered in terms of an elastic free energy that describes the stretching of the tail (from the shorter, disordered triacontanol to the all-trans triacontanoic acid). ${ }^{17-19}$ Considering only the hydrogen bonding and the elastic energy,

$$
\frac{f}{k T}=-\frac{\epsilon \rho^{2}}{2}+\rho \frac{3\left(h-h_{\mathrm{o}}\right)^{2}}{2 N b^{2}}
$$

where the first term of the free energy per area $f$ (normalized by the thermal energy $k T$ ) is the energy due to hydrogen bonding ( $\epsilon$ is the H-bonding energy, measured in units of $k T$ times the area of a close-packed headgroup $A$, and $\rho$ is the number of headgroups per interfacial area). The second term accounts for tail stretching ( $h$ is the thickness of the layer, $h-h_{\mathrm{o}}$ is the stretching length, $N$ is the number of gauche defects, and $b$ is the average distance between gauche defects). Assuming that the tails are incompressible leads to $\rho=h / V$, where the constant $V$ is the volume of the tail. Substituting $\rho=h / V$ into eq 1 and minimizing the free energy per area as a function of thickness, $h$ leads to the equilibrium thickness $h^{*} \approx h_{\mathrm{o}}+\epsilon N b^{2} / 3 V$. This indicates that the monolayer thickness increases (the tail stretches) as the attractive interaction $\epsilon$ becomes stronger. We take $h_{\mathrm{o}}$ to be the thickness of the disordered triacontanol monolayer, that is, the monolayer thickness for $\epsilon=0$. If we let $\epsilon=8 A$ and $N=5 \mathrm{H}$-bonds distributed over half the tail (so $b$ $=L /[2 *(N+1)]$, where $L$ is the length of the all-trans chain), and take $V=L A$, then the layer thickness increases by $4 \AA$, similar to the experimental observation (Table 1).

In conclusion, at the water-vapor interface, $n$-alkanols and $n$-alkanoic acids of sufficient tail length form monolayer phases containing essentially all-trans tails. ${ }^{20}$ However, at the water- hexane liquid-liquid interface, the molecular ordering is different, with long-chain $n$-alkanols, from 20 up to 30 carbons long, forming monolayer phases with disordered tails., ${ }^{5,6}$ By studying triacontanoic acid monolayers at the water-hexane interface, we have demonstrated that a small change in the headgroup structure, which alters the hydrogen bonding interactions, leads to a large conformational change from disordered to ordered tailgroups.

Acknowledgment. M.L.S. thanks John Marko for suggesting eq 1. Financial support is gratefully acknowledged from NSF DMR (for M.L.S.), NSF BES and NSF NSEC for Directed Assembly of Nanostructures (for S.G.), NSF Chemistry (for ChemMatCARS), and DOE (for ChemMatCARS, Brookhaven, and Argonne National Laboratories).

\section{References and Notes}

(1) Safran, S. A. Statistical Thermodynamics of Surfaces, Interfaces, and Membranes; Addison-Wesley Publishing Co.: Reading, MA, 1994.

(2) Richmond, G. L. Annu. Rev. Phys. Chem. 2001, 52, 357.

(3) Schlossman, M. L. Curr. Opin. Colloid Interface Sci. 2002, 7, 235

(4) Watry, M.; Richmond, G. L. J. Am. Chem. Soc. 2000, 122, 875

(5) Tikhonov, A. M.; Schlossman, M. L. J. Phys. Chem. B 2003, 107, 3344.

(6) Tikhonov, A. M.; Pingali, S. V.; Schlossman, M. L. J. Chem. Phys. 2004, 120, 11822

(7) Zhang, Z.; Mitrinovic, D. M.; Williams, S. M.; Huang, Z.; Schlossman, M. L. J. Chem. Phys. 1999, 110, 7421.

(8) Water was produced by a Barnstead Nanopure system. $n$-Hexane (Aldrich-Sigma) was purified by passing it through activated alumina in a chromatography column. Triacontanoic acid and triacontanol (Fluka) were recrystallized twice at room temperature from an oversaturated solution in $n$-hexane that was originally prepared at $60{ }^{\circ} \mathrm{C}$.

(9) Gaines, G. L., Jr. Insoluble Monolayers at Liquid-Gas Interfaces; Interscience Publishers: New York, 1966.

(10) Spink, J. A. J. Colloid Sci. 1963, 18, 512

(11) Schlossman, M. L.; Synal, D; Guan, Y; Meron, M.; SheaMcCarthy, G.; Huang, Z.; Acero, A.; Williams, S. M.; Rice, S. A.; Viccaro, P. J. Rev. Sci. Instrum. 1997, 68, 4372.

(12) Tidswell, I. M.; Ocko, B. M.; Pershan, P. S.; Wasserman, S. R.; Whitesides, G. M.; Axe, J. D. Phys. Rev. B 1990, 41, 1111

(13) Small, D. M. The Physical Chemistry of Lipids; Plenum: New York, 1986.

(14) Schlossman, M. L.; Schwartz, D. K.; Kawamoto, E. H.; Kellogg, G. J.; Pershan, P. S.; Ocko, B. M.; Kim, M. W.; Chung, T. C. In Materials Research Society Proceedings - Macromolecular Liquids; Safinya, C. R., Safran, S. A., Pincus, P. A., Eds.; Materials Research Society: Boston, MA, 1990; Vol. 177, p 351.

(15) Kalra, A.; Garde, S.; Hummer, G. Proc. Natl. Acad. Sci. U.S.A. 2003, 100, 10175

(16) Marcelja, S. Biochim. Biophys. Acta 1974, 367, 165

(17) Alexander, S. J. Phys. (Paris) 1977, 38, 983.

(18) de Gennes, P. G. Scaling Concepts in Polymer Physics; Cornell University Press: Ithaca, NY, 1979.

(19) de Gennes, P. G. Macromolecules 1980, 13, 1069.

(20) Kaganer, V. M.; Mohwald, H.; Dutta, P. Rev. Mod. Phys. 1999 71,779 .

(21) Berendsen, H. J. C.; Postma, J. P. M. J. Chem. Phys. 1984, 81, 3684 .

(22) Jorgensen, W. L.; Chandrasekhar, J. J. Chem. Phys. 1983, 79, 926.

(23) Cornell, W. D.; Cieplak, P.; Bayly, C. I.; Gould, I. R.; Merz, K. M. J.; Ferguson, D. M.; Spellmeyer, D. C.; Fox, T.; Caldwell, J. W. Kollman, P. A. J. Am. Chem. Soc. 1995, 117, 5179.

(24) Ryckaert, J. P.; Ciccotti, G. J. Comput. Phys. 1977, 23, 327.

(25) Darden, T.; York, D.; Pedersen, L. J. Chem. Phys. 1993, 98, 10089. 